\title{
STRIDE-CYCLE INFLUENCES ON GOAL-DIRECTED HEAD MOVEMENTS MADE DURING WALKING
}

Brian T. Peters ${ }^{1,2}$, Richard E.A. van Emmerik ${ }^{2}$, and Jacob J. Bloomberg ${ }^{3}$

${ }^{1}$ Neuroscience Laboratories, Wyle Life Sciences, Houston, TX, USA

${ }^{2}$ Department of Exercise Science, University of Massachusetts, Amherst, MA, USA

${ }^{3}$ Neuroscience Laboratories, NASA/Johnson Space Center, Houston, TX, USA

Subtitle: Head Movements Made While Walking

Correspondence:

Brian T. Peters

Wyle Life Sciences

1290 Hercules Dr. Suite 120

Houston, TX 77058, USA

Phone: (281) 244-6574; Fax: (281) 244-5734

e-mail: bpeters@ems.jsc.nasa.gov 


\begin{abstract}
$\underline{\text { Abstract }}$
Horizontal head movements were studied in six subjects as they made rapid horizontal gaze adjustments while walking. The aim of the present research was to determine if gait-cycle events alter the head movement response to a visual target acquisition task. Gaze shifts of approximately $40^{\circ}$ were elicited by a step change in the position of a visual target from a central location to a second location in the left or right horizontal periphery. The timing of the target position change was constrained to occur at $25,50,75$ and $100 \%$ of the stride cycle. The trials were randomly presented as the subjects walked on a treadmill at their preferred speed (range: 1.25 to $1.48 \mathrm{~m} / \mathrm{s}$, mean: $1.39 \pm 0.09 \mathrm{~m} / \mathrm{s}$ ). Analyses focused on the movement onset latencies of the head and eyes and on the peak velocity and saccade amplitude of the head movement response. A comparison of the group means indicated that the head movement onset lagged the eye onset ( 262 ms versus 252 $\mathrm{ms}$ ). The head and eye movement onset latencies were not affected by either the direction of the target change nor the point in the gait cycle during which the target relocation occurred. However, the presence of an interaction between the gait cycle events and the direction of the visual target shift indicates that the peak head saccade velocity and head saccade amplitude are affected by the natural head oscillations that occur while walking.
\end{abstract}

Key words: head movement, target acquisition, locomotion, eye-head coordination, vision 
Introduction

Visual fixation of targets peripheral to the progression of travel is common as we walk, yet gaze adjustments made during locomotion have not been systematically studied. When these gaze adjustments are sufficiently large, a head rotation must accompany the eye movements to achieve the goal. Little is known about the interaction between these goal-directed head movements and the periodic head translations and rotations that naturally accompany locomotion. Reports vary regarding the magnitude of these periodic head movements that occur while walking, but the phase relationship between these movements and ongoing gait cycle is consistent ${ }^{1-8}$. In the sagittal plane for example, the head pitches upward as the body translates down during each step. Yaw head movements counter the lateral translations of the body in a similar way. Based on studies using passive rotations of the body, it is believed that these movements are the result largely of reflexive mechanisms ${ }^{9,10}$. Regardless of the control mechanism (i.e. reflexive, voluntary, or passive inertial) these head movements imply the presence of modulating levels of neck muscle activation and vestibular stimulation.

The effects of this modulation on goal-directed head movements are unknown. Bent and colleagues found differences in lower body gait parameters that were dependent on when in the gait cycle a vestibular stimulus was given ${ }^{12}$. A head saccade made while walking could impart a similar vestibular disturbance. The results of a study comparing the effects of voluntary and unexpected head turns while walking, led Vallis and Patla to conclude that the central nervous system partially nullifies the sensory input created by voluntary head turns ${ }^{13}$. In addition to this proposed use of efferent copy to minimize the vestibular disturbance, a strategy of triggering head movements to coincide with phases of the gait cycle where the vestibular information is less important could also be used. Such a strategy would affect the timing of the head movement response. Differences in the response timing may also result from interactions with other ongoing activities. Lajoie et al. showed that reaction time was dependent on when in the gait-cycle the stimulus was presented ${ }^{11}$. The authors attributed this result to varying levels of attentional demand across the gait cycle. 
In addition to possible changes in the initiation of a response, the process of superimposing a voluntary head movement on the naturally occurring motions may affect the dynamics of the head movement response. The eye-head coordination during gaze re-fixations has.been studied extensively in seated subjects, but this behavior has not been studied in walking subjects. Changes in the head movement dynamics could impose limitations on the total response time (i.e. reaction time plus movement time) and could result in final head positions that leave the eyes at orbital eccentricities that are sub-optimal for visual acuity. Through measures of movement onset latency and head movement kinematics, the goal of the present research was to assess the effects of gait cycle events on head movements made to acquire targets in the visual periphery.

\section{Materials and Methods}

Subjects

Six healthy males in the age range from 26 to 35 (mean 31 years \pm 4 ) served as subjects for this study. None of the subjects had complaints of neck soreness at the time of the test and none had any history of vestibular disease. The experiment protocol was approved by the University of Massachusetts' Human Subjects Review Committee and all subjects gave their informed consent prior to participation.

\section{Testing Conditions}

\section{Walking Speed}

The subjects, all wearing a similar type jogging shoe, were tested as they walked on a motordriven treadmill (Accumill P, Pacer Fitness Systems, Inc., Irving, TX) at their preferred walking speed. The subject-specific speeds were established using an interactive method defined in Holt et al ${ }^{14}$ which resulted in speeds ranging from 1.25 to $1.48 \mathrm{~m} / \mathrm{s}$ (mean $1.39 \pm 0.09 \mathrm{~m} / \mathrm{s}$ ).

\section{Visual Targets}

Visual targets were presented on a rear-projection screen (Da-Lite Screen Company, Inc., Warsaw, Indiana) that was placed between the subject and an LCD projector (Sharp, Model: XGE670U, Mahwah, New Jersey). The screen was perpendicular to the walking direction at a distance 
of $\sim 110 \mathrm{~cm}$ from the subject's nasal bridge. The targets, consisting of alpha-numeric characters, were controlled by custom software (LabVIEW; National Instruments Corp., Austin, TX.). When presented directly in front of the subject, the visual targets subtended a visual angle of $\sim 1.3^{\circ}$.

During each of the 14-second walking trials, the visual target was initially presented in the center of the subject's field-of-view at a height that was perceived by them to be eye level. The presented targets were randomly selected from a "pool" that contained 24 upper-case characters ( $\mathrm{O}$ and I were excluded) and 8 numerals ( 0 and 1 were omitted). With the exception of the first target to appear in the lateral position, which was visible for $900 \mathrm{~ms}$, the display duration for the sequentially displayed characters was assigned randomly using the finite set of times that fell at 50 ms intervals from 400 to $700 \mathrm{~ms}$.

Targets in the lateral positions were restricted to the pool of 24 letters and presented at the same height as the central target but at positions that were $1 \mathrm{~m}$ to the left or right of it. A gaze compensation angle of $\sim \pm 40^{\circ}$ was required to visually fixate the lateral targets that continued to be displayed until the end of the trial. At the conclusion of each trial the subject 's recollection of the number of numerals that appeared in the central position, as well as the first letter seen in the lateral position, was compared to the actual values. While this information was used to establish an inclusion criterion for acceptable data trials, the primary intent of requiring this from the subject was to motivate them to concentrate on the task.

The timing of the target change from the central position to the lateral position was one of the critical aspects of this investigation. The stimulus-generating program used a signal produced by a roller switch secured to the lateral edge of the heel of the right shoe to control this change. Measured from the right heel strike, the target position changes were restricted to four phases, or quadrants, corresponding to $25,50,75$ and $100 \%$ of the stride cycle.

The two lateral positions and four stride quadrants created eight possible test conditions, which were each repeated three times per subject. The order of the twenty-four trials was randomized prior to the start of data collection. 


\section{$\underline{\text { Standing test }}$}

Although a comparison between walking and standing conditions was not the intended focus of this investigation, relatively long reaction time latencies during the walking task prompted us to repeat the task during a subsequent test session. Five of the six subjects repeated the gaze refixation task as they stood on the non-moving treadmill belt.

\section{Measurement Systems}

Kinematic and analog variables were collected at $240 \mathrm{~Hz}$ using the Qualisys motion analysis system (Qualisys, Inc., Glastonbury, CT.). The system monitored the positions of seven retroreflective markers affixed to a light-weight headband $(183 \mathrm{~g})$ and a torso harness. The relative position of a virtual marker placed on the subject's nasal bridge was determined prior to the start of the data collection. This information allowed the location of that landmark to be calculated throughout each of the data trials.

The analog signals consisted of two TTL outputs that were used to indicate right heel strike and the timing of visual target position change and horizontal eye movements. The eye movements were obtained using standard DC Electrooculography (EOG) techniques.

\section{Data Processing}

\section{Head Yaw Calculation}

A line segment connecting the virtual nasal bridge marker and the marker placed on the occipital region of the head was used to calculate the yaw movements of the head. Because this line segment deviated only slightly above and below $\left(\sim \pm 2^{\circ}\right)$ the transverse plane while walking any angle calculation errors introduced by out-of-plane motions were minimal.

\section{Movement Onset Determination}

The onset of the saccadic movements for both the head and eyes was considered to be the point at which their velocity trajectories deviated from $\mathrm{a} \pm 1$ SD band around the average per-stride trajectory. A fourth-order, zero-lag Butterworth filter was applied to the raw position data prior to 
calculating the velocities with a 3-point central difference algorithm. The per-stride average trajectories and standard deviations (SD) were calculated for each trial using the first six complefe strides, each of which had been linearly interpolated to 250 points. Because the $5 \mathrm{~Hz}$ filter applied to the data prior to determining the average trajectory significantly attenuated the sharpness of the saccadic movement, the epoch containing the saccade was filtered using $15 \mathrm{~Hz}$ cut-off. The point at which this signal deviated from the average trajectory was automatically determined using customwritten software (MATLAB; The Mathworks, Inc., Natick, MA). An operator who was sufficiently blinded from knowing the condition of the trial being displayed, visually inspected each trial and was able to manually input the appropriate position if the location of the automatically determined movement onsets was incorrect.

\section{Peak Yaw Head Velocity}

The peak yaw head velocity was determined to be the maximum value observed in the filtered ( $15 \mathrm{~Hz}$ cutoff) yaw velocity. This value was also visually inspected from the time series data.

\section{Yaw Head Saccade Amplitude}

Variability in the stride-to-stride trajectory of the yaw head position prevented the "offset" of the saccade from being reliably determined. In lieu of using the offset to determine the amplitude of the head saccade, it was calculated to be the difference between the average yaw position of the head during the first complete stride after the saccade and the average position of the last complete stride before the saccade.

\section{Statistical Analysis}

Prior to performing any statistical analyses, data from 5 of the 120 trials were excluded from the data set because the target position change occurred outside a $\pm 5 \%$ window of the desired stride position (i.e. 20 - $30 \%$ for the $25 \%$ condition). Another 19 trials were eliminated because the subjects did not accurately identify the first letter that appeared in the lateral position. This was usually the result of a response that was sufficiently delayed due to either starting the movement in the opposite direction or because of a lack of attention. An ANOVA using a split-plot factorial 
design was used for the statistical analyses. The ANOVA model contained tests for main effect differences between SUBJECTS, TRIALS, target step DIRECTION, and gait-cycle QUADRANT. It also included a test for a DIRECTION x QUADRANT interaction that was nested within the SUBJECTS. The test for TRIALS effects was included to make the analysis complete, but as would be expected given the randomized presentation of the 24 trials this comparison showed no effect. Therefore, it is not included in the discussion of the results.

\section{$\underline{\text { Results }}$}

\section{Basic Head and Eye Motions in Yaw}

While walking and attending to the visual target, the yaw head motion of all of the subjects had an oscillatory nature that repeats itself with each stride (i.e. heelstrike to heelstrike of the same foot). This oscillation is present during gaze fixation of both the central and lateral target locations (see Figure 1). The inset in Figure 1 shows the per-stride average head yaw trajectory for the central target portion of the trial. The desired $25,50,75$, and $100 \%$ points for the possible target relocation times are depicted on the plot.

\section{Insert Figure 1 here}

In all subjects, the medio-lateral position of a marker located near the seventh cervical vertebra maintained an anti-phase relationship with the head yaw signal. Across all of the data trials the peak-to-peak amplitude of the lateral trunk translation was $4.9 \pm 1.0 \mathrm{~cm}$ (mean $\pm 1 \mathrm{SD}$ ) (range: 3.1 to $5.6 \mathrm{~cm}$ ) and the peak-to-peak amplitude of the head yaw rotation was $3.2 \pm 1.2$ degrees (range: 2.1 to 5.1 degrees). Four of the six subjects also had horizontal eye movements that clearly showed a similar oscillatory pattern that maintained an anti-phase relationship with the head yaw rotation (see Figure 1), indicating that when the head rotated to the left, the eyes rotated to the right. This type of coordination could be consistent with a natural head orientation projection that crosses the standing line of sight between the subject and the visual target ${ }^{15}$. In this scenario, the counter- 
rotation of the eyes is necessary because the head rotation over-compensates for the lateral translation of the trunk ${ }^{8,16}$.

\section{Reaction Time Variables}

With subject means ranging from 209 to $288 \mathrm{~ms}$ (group mean: $252 \mathrm{~ms}$ ), the horizontal eye movement onset latencies varied significantly between subjects $(p<0.001)$. This was also true for the latency of the onset for the head yaw movement (range: 234 to $290 \mathrm{~ms}$; group mean: $262 \mathrm{~ms}$ ). The group means suggest that the onset of the eyes preceded the head movement onset, but this was only clearly evident for half of the subjects (see Figure 2). A presentation of the p-values obtained for each of the dependent variables across all experimental manipulations is provided in Table 1. It is evident from these data that neither of the reaction time variables were affected by the experimental manipulations

\section{Insert Figure 2 here}

Insert Table 1 here

\section{Head Saccade Dynamics}

The saccade amplitude and peak velocity of the head yaw signal were measured to provide an assessment of head saccade dynamics. Across all subjects the mean peak velocity values ranged from $64-172 \%$ and the amplitude of the head saccade ranged from $3-25^{\circ}$. Each of these measures illustrate that the head dynamics of the saccade response differed between subjects $(\mathrm{p}<0.001$ for each). A statistically significant interaction between the direction of the required gaze re-fixation and the point in the gait cycle at which the target changed locations was also observed in each ( $\mathrm{p}=0.015$ and $\mathrm{p}=0.021$ for peak velocity and saccade amplitude, respectively). The presence of this interaction, clearly visible in the group means presented in Figure 3, indicates that stride cycle parameters can influence the head movements made to acquire visual targets while walking. 
A reason for this interaction becomes apparent when inspecting a subject's head yaw velocity signals from the 25 and $75 \%$ conditions (see Figure 4). A comparison of the peak velocity amplitudes between the two conditions shows that movements made to fixate a target in the left periphery achieve higher magnitudes in the $75 \%$ condition than those in the $25 \%$ condition. The opposite is true for rightward saccades. The slope of the head velocity signal just prior to the onset of the movement shows the direction of the head's rotational acceleration at the time that the movement is initiated. An upward slope indicates that the head is accelerating to the left. When the visual target appears in the periphery to the side that the head is naturally accelerating, the accompanying head movement saccade achieves a higher peak velocity. When required to stop and accelerate in the opposite direction the magnitude of the peak velocity of the saccade is lower. These results indicate that the natural motions of the head that occur while walking affect the dynamics of the head saccade.

\section{Insert Figure 4 here}

\section{Discussion}

The results presented here suggest that the head movement responses to visual target position changes can be influenced by the phase of the gait cycle during which the target relocation takes place. Differences were present in the peak head velocity and head saccade amplitude signals, but the movement onset latencies of the eye and head movements appeared to be unaffected by the experimental manipulations.

The fact that the reaction time variables (i.e. the eye and head movement onset latencies) were unaffected by the experimental conditions of this walking paradigm is in contrast with previously reported results of reaction time tasks performed while walking ${ }^{11,17,18}$. The previous 
studies reported longer reaction times when in the single-support phase of walking when compared to the double-support phase. The authors attributed this difference to a modulation in attentional demands during the stride cycle. The disparate results between these studies and the current one may be linked to the different experimental tasks. The subjects in the previous studies were required to respond verbally to an auditory stimulus whereas a visual stimulus and non-verbal response were used in the present study. Although the modality of a sensory stimulus can affect reaction times, it seems more likely that the cognitive demand of the required response rather than the perception of the stimulus is the reason that a similar result was not present in the current paradigm. A visual task that requires a higher cognitive demand prior to making the response, such as an anti-saccade visual task may be able to validate this assumption.

A rapid change in head position accompanies some gaze adjustments and this change creates afferent vestibular signals. By observing the effects of vestibular stimulation on lower-body gait parameters, Bent et al. showed that some portions of the gait cycle are more susceptible to vestibular stimulation than others ${ }^{19}$. The initiation of the head movement responses in this present task could have been triggered to avoid these susceptible periods. The similar movement onset latencies of the gaze response, including the head movement response, observed in the results presented here provides evidence that the gaze adjustments are not constrained to occur at the periods of lesser vestibular influence. This lack of phase dependency however, does not imply a lack of coordination between the visual and locomotor tasks. Indeed a study comparing the effects of voluntary and unexpected head turns during walking provides evidence that the perturbations to the cyclical vestibular afferents are at least partially nullified by the central nervous system during a voluntary head turn ${ }^{13}$. This nullification minimizes potential threats to gait stability regardless of when in the gait cycle the movement occurs. It stands to reason that the visual acquisition of targets not be constrained by gait cycle events. The potential importance of the information obtained through a saccadic eye/head re-fixation may be very high. Delays in obtaining this information could be detrimental. 
Although a dependency on the phase of the gait cycle was not evident in the onset latencies of the response, the same cannot be said for the peak head saccade velocity and the head saccade amplitude. Evidence for a gait-cycle dependent influence on saccadic gaze shifts is found in the statistical interactions that occur in these variables. The initial static head position has been shown to influence the dynamics of the head movement response ${ }^{20}$, but the data presented in Figure 4 provide evidence that the direction that it is accelerating can also influence the movement characteristics. Interesting questions can be raised regarding the interaction between the underlying neuromuscular mechanisms responsible for these natural movements and those necessary to make the head saccade. The differences in head saccade dynamics presented here could be the result of an open-loop neural command that generates the same change in neck muscle activation regardless of the head motions that are occurring at the time of the movement onset. Such a system would simplify the effects on the descending neural commands, but may do so at the expense of accurate gaze orientation. Empirical studies that have a more direct measure of the visual systems abilities during locomotion may provide insight into the optimization parameters that govern the interaction between obtaining visual information while walking and walking itself.

While it is reasonable to assume that walking imposes a different set of constraints on vision relative to a non-locomotory condition, follow-up data collected to address methodological questions in the current protocol indicate that the new limits may not be more restrictive than those in a static condition. The head movement onset latencies for all of the subjects in the current protocol fell either beyond, or in the high range, when compared to data gathered from seated subjects using a similar target displacement amplitude ${ }^{21}$. To rule out experimental factors that may have been the cause of this result, a subset $(n=4)$ of the original subjects repeated the visual manipulations while standing on the non-moving treadmill. Interestingly, all of the subjects had longer movement onset latencies while standing compared to their walking trials. From this result, we were able to conclude that it was likely the cognitive demands of counting and remembering the number of numerals seen while the target remained in the central field of view that caused the higher latency times. Most target acquisition studies use point light displays for the visual targets. The 
appearance of an enhanced ability to perceive a visual target in a new location while walking requires a protocol that is specifically designed to address the issue, but this preliminary result indicates that locomotion may not be in all cases a detriment to visual performance. From the data presented here, future similar studies that focus only on the onset latencies can be conducted without regard for gait cycle influences. However, those that include investigations of head movement dynamics and even overall response times should consider the effects of gait events in their design. 


\section{Acknowledgement}

This research and the preparation of this article were supported by a grant from the NASA Graduate Student Researchers Program awarded to Brian T. Peters (NGT 5-28932). The results presented here were also submitted to the Graduate School of the University of Massachusetts in partial fulfillment of the requirements of the Master of Science degree program of Mr. Peters. R.E.A. van Emmerik was supported by grant \#RG99-0097 from the Whitaker Foundation and Mr. Peters and Dr. Bloomberg were both supported through NASA Cooperative Agreement NCC9-58 with the National Space Biomedical Research Institute. 


\section{BIBLIOGRAPHY}

1. Pozzo $T$, Berthoz A, Lefort L. Head kinematic during various motor tasks in humans. In: Allum JHJ, Hulliger M, eds. Progress in Brain Research. Vol 80: Elsevier Science Publishers; 1989:377-383.

2. Pozzo T, Berthoz A, Lefort L. Head stabilization during various locomotor tasks in humans. I. Normal subjects. Exp Brain Res. 1990;82(1):97-106.

3. Pozzo T, Berthoz A, Vitte E, Lefort L. Head stabilization during locomotion. Acta Otolaryngol (Stockh). 1991;Suppl 481:322-327.

4. Keshner EA, Peterson BW. Multiple Control Mechanisms Contribute to Functional Behaviors of the Head and Neck. In: Berthoz A, Graf W, Vidal PP, eds. The Head-Neck Sensory System. New York: Oxford University Press; 1992:381-386.

5. Bloomberg JJ, Reschke MF, Huebner WP, Peters BT. The effects of target distance on eye and head movement during locomotion. Ann N Y Acad Sci. 1992.

6. Berthoz A, Pozzo T. Head and Body Coordination during Locomotion and Complex Movements. Interlimb Coordination: Neural, Dynamical, and Cognitive Constraints: Academic Press, Inc; 1994.

7. Hirasaki E, Moore ST, Raphan T, Cohen B. Effects of walking velocity on vertical head and body movements during locomotion. Exp Brain Res. 1999;127(2):117-130.

8. Moore ST, Hirasaki E, Cohen B, Raphan T. Effect of viewing distance on the generation of vertical eye movements during locomotion. Exp Brain Res. 1999;129(3):347-361. 
9. Keshner EA, Cromwell RL, Peterson BW. Mechanisms controlling human head stabilization. II. Head-neck characteristics during random rotations in the vertical plane. $J$ Neurophysiol. 1995;73(6):2302-2312.

10. Keshner FA, Peterson BW. Mechanisms controlling human head stabilization. I. Headneck dynamics during random rotations in the horizontal plane. J Neurophysiol. 1995;73(6):2293-2301.

11. Lajoie Y, Teasdale N, Bard C, Fleury M. Attentional demands for static and dynamic equilibrium. Exp Brain Res. 1993;97(1):139-144.

12. Bent LR, Inglis JT, McFadyen BJ. When is Vestibular Information Important During Walking? J Neurophysiol. Sep 2004;92(3):1269-1275.

13. Vallis LA, Patla AE. Expected and unexpected head yaw movements result in different modifications of gait and whole body coordination strategies. Exp Brain Res. Jul 2004;157(1):94-110 .

14. Holt KG, Jeng SF, Ratcliffe R, Hamill J. Energetic cost and stability during human walking at the preferred stride frequency. JMot Behav. 1995;27(2):164-178.

15. Moore ST, Hirasaki E, Raphan T, Cohen B. The human vestibulo-ocular reflex during linear locomotion. Ann N Y Acad Sci. 2001;942:139-147.

16. Peters BT, Bloomberg JJ, Layne CS, McDonald PV, Huebner WP. Eye, head, and trunk phase relationships during treadmill locomotion while viewing visual targets at different distances. Paper presented at: Society for Neuroscience, 1996; Washington D.C.

17. Gage WH, Sleik RJ, Polych MA, McKenzie NC, Brown LA. The allocation of attention during locomotion is altered by anxiety. Exp Brain Res. Jun 2003;150(3):385-394. 
18. Lajoie $Y$, Teasdale N, Bard C, Fleury M. Upright standing and gait: are there changes in attentional requirements related to normal aging? Exp Aging Res. Apr-Jun 1996;22(2):185198.

19. Bent LR, McFadyen BJ, Inglis JT. Visual-vestibular interactions in postural control during the execution of a dynamic task. Exp Brain Res. Oct 2002;146(4):490-500.

20. Fuller JH. Head movement propensity. Exp Brain Res. 1992;92(1):152-164.

21. Fuller JH. Eye position and target amplitude effects on human visual saccadic latencies. Exp Brain Res. 1996;109(3):457-466. 
Table I

\begin{tabular}{|c|c|c|c|c|}
\hline & $\begin{array}{c}\text { Horizontal } \\
\text { EYE } \\
\text { Latency } \\
\end{array}$ & $\begin{array}{c}\text { Yaw HEAD } \\
\text { Latency }\end{array}$ & $\begin{array}{c}\text { Peak Yaw Head } \\
\text { Velocity }\end{array}$ & $\begin{array}{c}\text { Head Saccade } \\
\text { Amplitude }\end{array}$ \\
\hline SUBJECT & $<0.001$ & $<0.001$ & $<0.001$ & $<0.001$ \\
\hline OUADRANT. & 0.218 & 0.5.5. & 9249 & 0.525. \\
\hline DIRECTION & 0.436 & 0.664 & 0.610 & 0.311 \\
\hline SUBIOU ABXDIRECT) & 0.729. & 0.357 & 0.015 & 0.021 \\
\hline
\end{tabular}

Table I p-values for each of the primary dependent variables. 


\section{Figure Captions}

Figure 1 Time series data for one exemplar trial (left lateral target; 25\% quadrant). The solid bar on the heel-strike event trace represents the strides used to calculate the average trajectories. The inset shows the average head yaw trajectory for these strides. Circles on the averaged signal indicate the relative time points during which the visual target could have changed from the central to lateral positions.

Figure 2 Mean Eye (O) and Head (口) movement onset latencies (ms) for each subject. The error bars represent the standard deviations across trials. The filled symbols indicate the group means.

Figure 3 Peak Head Velocity (A) and Head Saccade Amplitude (B) data separated by target direction and gait cycle event. The group mean values for target step directions made to the left are shown as circles $(\mathbf{)}$, while those made to the right are represented by squares (a). Error bars represent \pm 1 standard error.

Figure 4 Head movement velocity trajectories from one subject for all of his trials for the 25 and 75\% quadrants. As indicated, positive value represented movements to acquire a target in the left periphery and negative values represent movements toward targets in the right. Time zero corresponds to the point at which the visual target disappeared from the central position and appeared in the periphery. The slopes of the velocity traces preceding the onset of the head saccade are highlighted by the parallel bars. 


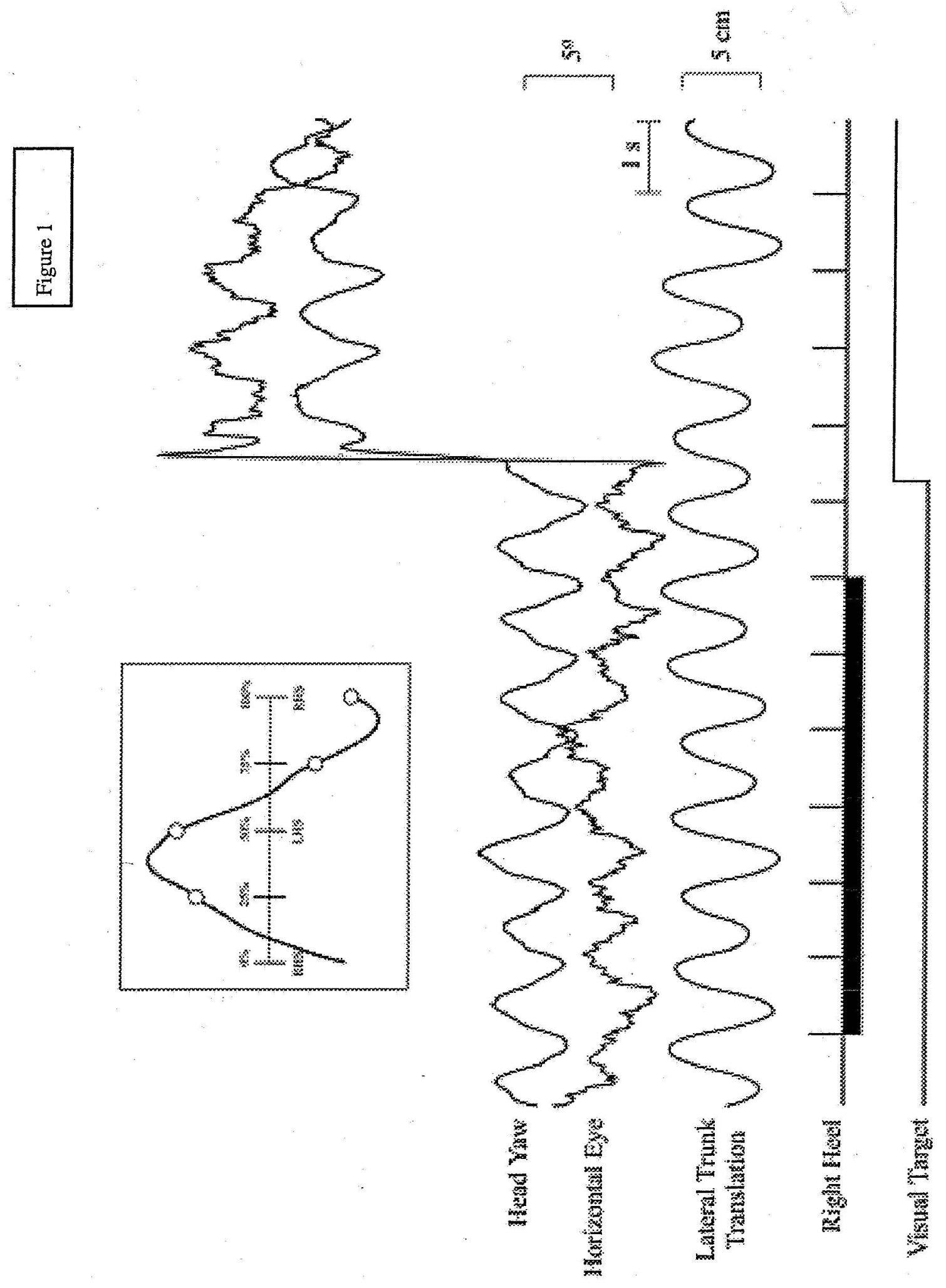




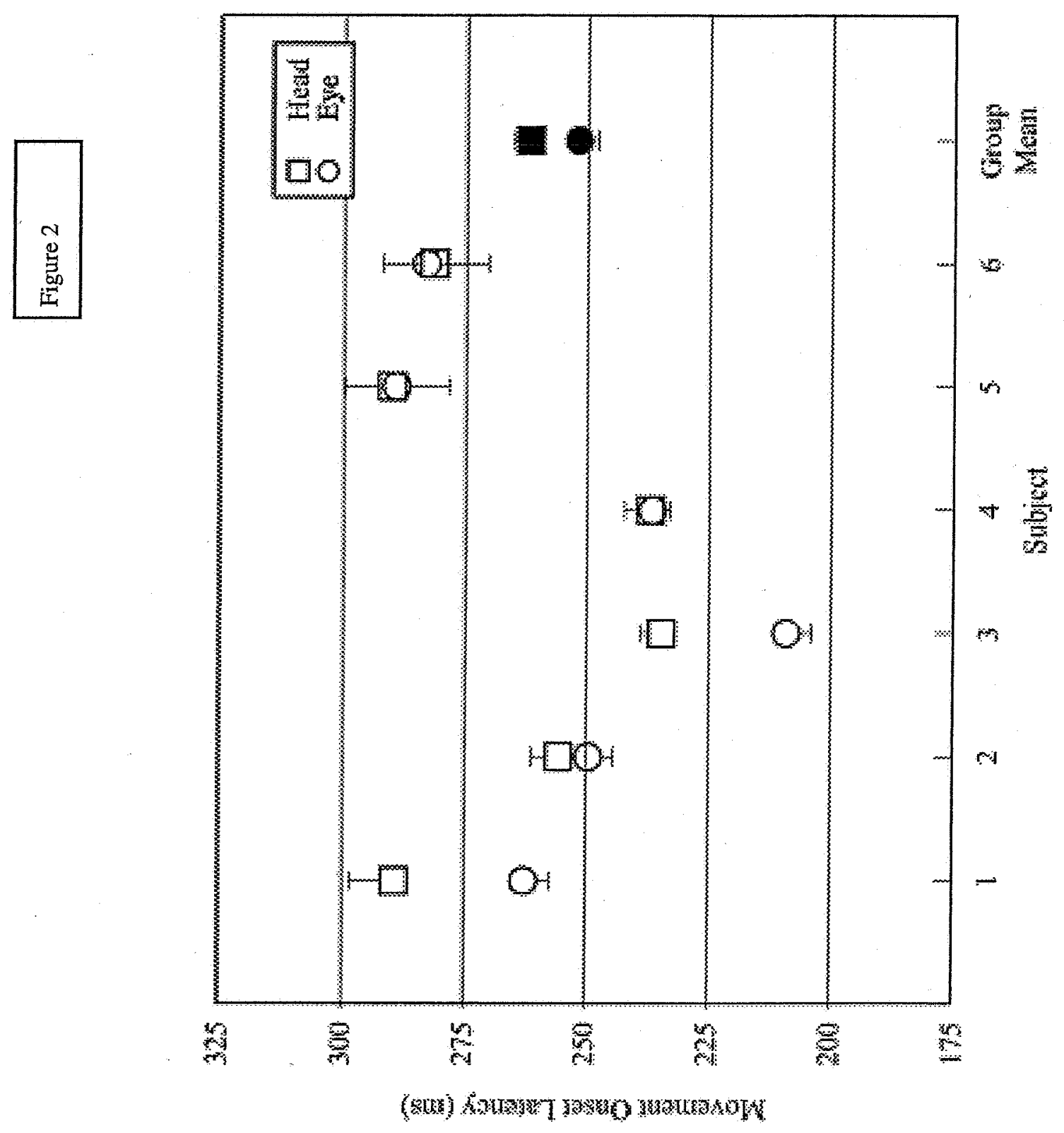



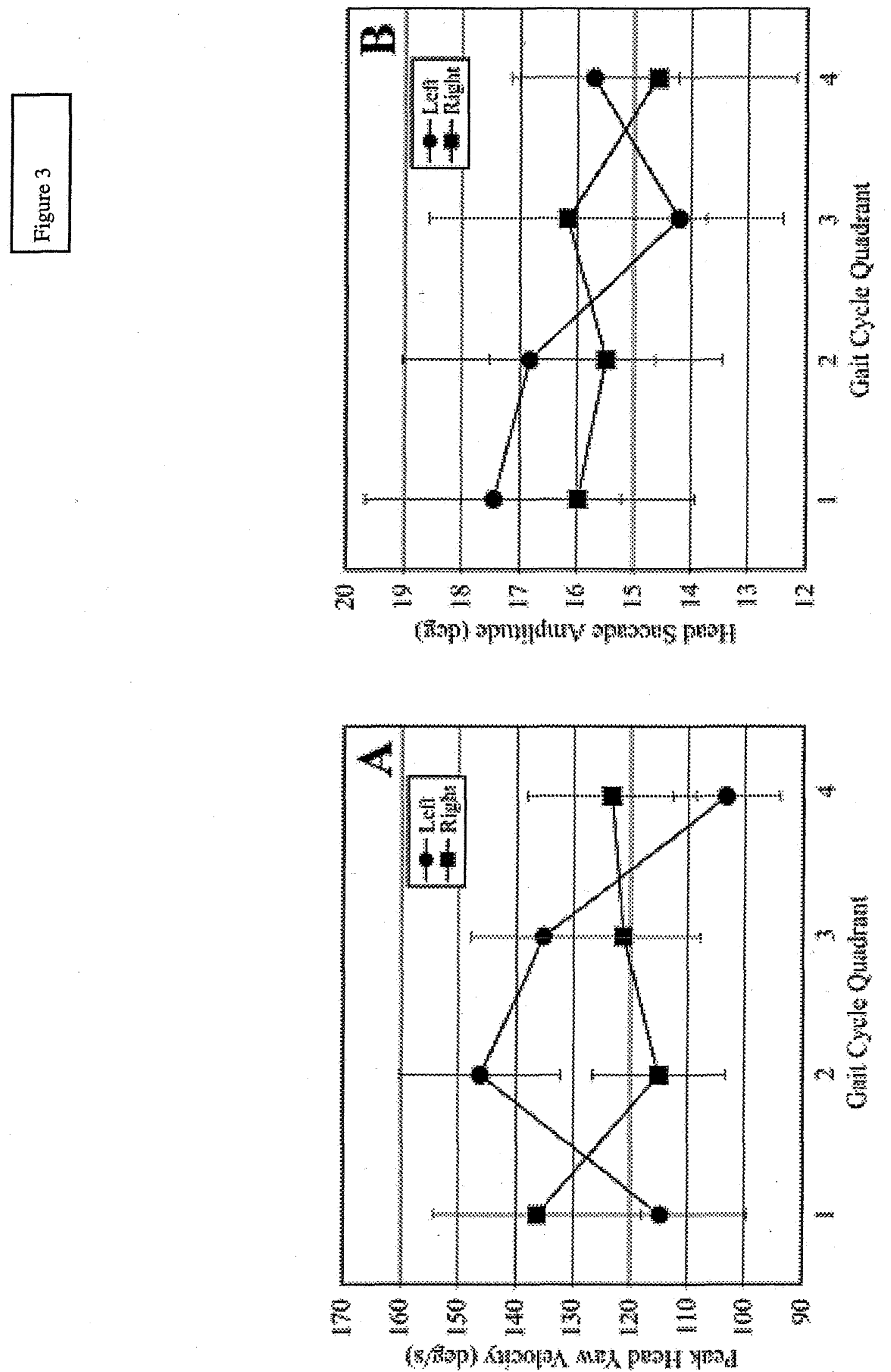

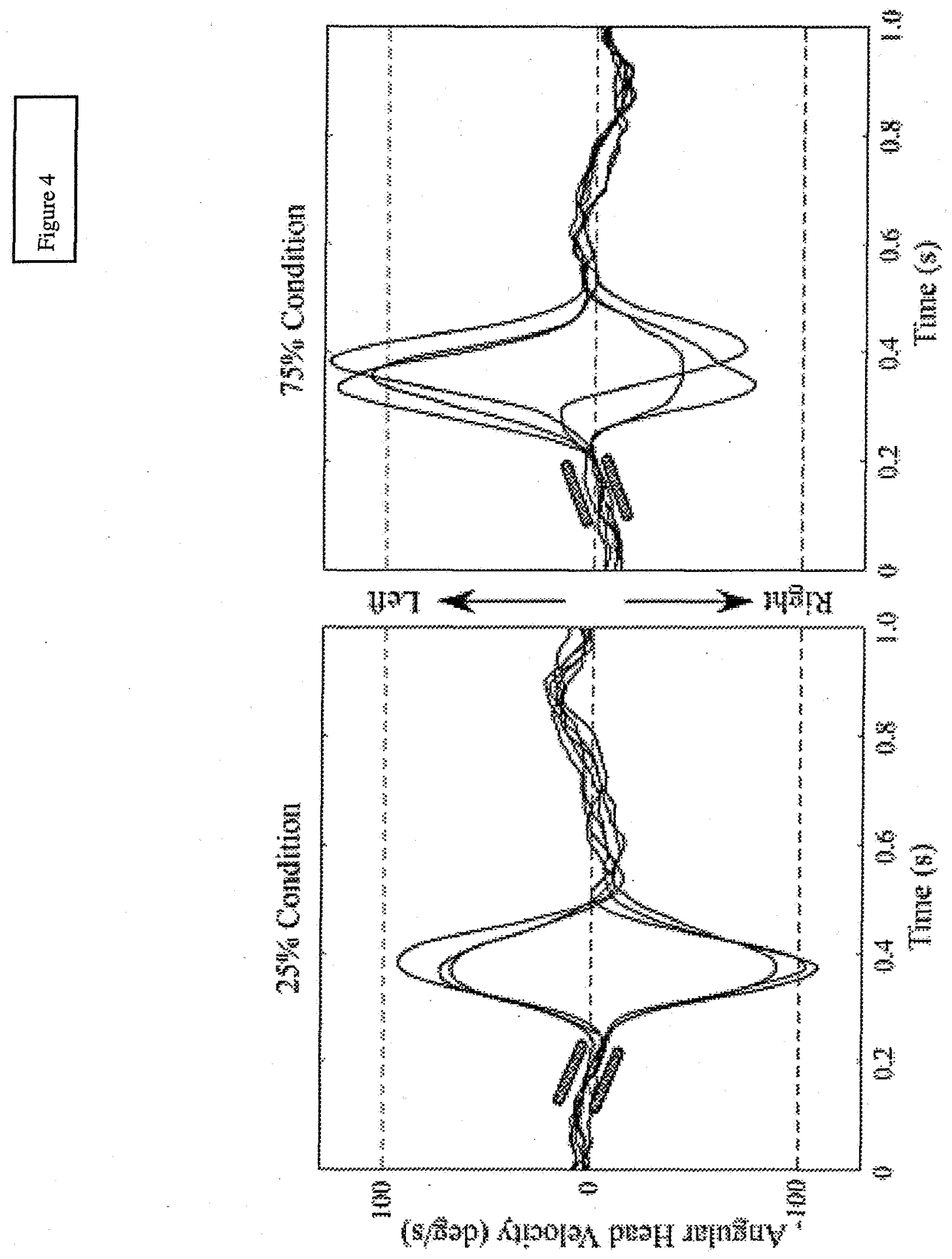